“ (C) 2017 IEEE. Personal use of this material is permitted. Permission from IEEE must be obtained for all other uses, in any current or future media, including

reprinting/republishing this material for advertising or promotional purposes, creating new collective works, for resale or redistribution to servers or lists, or reuse of any copyrighted component of this work in other works." 


\title{
A Comparison of Bidding Strategies for Online Auctions using Fuzzy Reasoning and Negotiation Decision Functions
}

\author{
Preetinder Kaur, Madhu Goyal, and Jie Lu
}

\begin{abstract}
Bidders often feel challenged when looking for the best bidding strategies to excel in the competitive environment of multiple and simultaneous online auctions for same or similar items. Bidders face complicated issues for deciding which auction to participate in, whether to bid early or late, and how much to bid. In this paper, we present the design of bidding strategies which aim to forecast the bid amounts for buyers at a particular moment in time based on their bidding behavior and their valuation of an auctioned item. The agent develops a comprehensive methodology for final price estimation which designs bidding strategies to address buyers' different bidding behaviors using two approaches: mamdani method with regression and negotiation decision functions. The experimental results show that the agents who follow fuzzy reasoning with regression approach outperform other existing agents in most settings in terms of their success rate and expected utility.
\end{abstract}

Index Terms-E-commerce, Fuzzy reasoning, Multiple linear regression, Online auctions, Negotiation decision functions, Software agents.

\section{INTRODUCTION}

$\mathrm{T}$ HE advent of electronic commerce has dramatically advanced traditional trading mechanisms, and online auction settings like eBay and Amazon have been emerged as a powerful tool for allocating goods and resources. Discovery of the new markets and the possibilities opened by online trading has heightened the sellers' and buyers' interest. In recent years, online auctions have become a widely recognised paradigm of item exchange, offering traders greater flexibility in terms of both time and geography. In online auction commerce, traders barter over products, applying specific trading rules over the Internet which support different auction formats. Common online formats are English, Dutch, First-price sealed-bid and Second-price sealed-bid auctions [1, 2].

Bidders in this marketplace face difficulties when looking for the best bidding strategies to win the auction. Moreover, there are commonly many auctions selling the desired item at any one time. Deciding which auction to participate in, whether to bid early or late, and how much to bid are very complicated issues for bidders [3,4]. The difficult and timeconsuming processes of analysing, selecting and making bids and monitoring developments need to be automated in order to assist buyers with their bidding.

The emergence of software agent technology has created an innovative framework for developing online auction mechanisms. Because of their extraordinary adaptive capabilities and trainability, software agents have become an integral component of online trading systems for buying and selling goods. These software agents represent expert bidders or sellers to fulfil their requirements and pursue their beliefs, and are consequently trained to achieve these aims. Software agents can perform various tasks like analysing the current market to predict future trends, deciding bid amounts at a particular moment in time, evaluating different auction parameters and monitoring auction progress, as well as many more. These negotiating agents outperform their human counterparts because of the systematic approach they take to managing complex decision-making situations effectively [5]. This creates more opportunities for expert bidders and sellers to maximise satisfaction and profit. Software agents make decisions on behalf of the consumer and seek to guarantee that items are delivered to the buyer's preferences. To function well, these agents must have prior knowledge of the auction's features, whether these are certain or uncertain.

eBay is one of the major global online marketplaces and currently the biggest consumer-to-consumer online auction site. Founded in 1995, eBay Inc. has attracted over 112 million active users and gained a net revenue of $\$ 14.1$ billion for 2012 . eBay does not, however, actually sell any goods that it owns; it only makes the process of displaying and selling goods easier by facilitating the bidding and payment processes. In virtual terms, eBay provides a marketplace where buyers and sellers meet and transact. eBay is a great source of high quality data as it keeps detailed records of completed auctions, and this data has been used extensively by researchers to solve research issues involving online auctions [6-12].

eBay-style auctions adopt the English auction format, except with regard to the payment of the winning bid [2]. In eBay auctions, the winner is the bidder with the highest value bid, but instead of paying his own bid, he pays the secondhighest bid plus the amount of one bid increment. Bidders in these auctions do not, however, bid their maximum valuation of the item offered. This is either because they do not grasp that they should do so or they simply have trouble figuring out what their maximum valuation is. These bidders are typically afraid of winning the auction at a price above the true value of the item, a phenomenon sometimes known as 'the-winner's 
curse'. This problem occurs because the bidder lacks information about the true value of the item. In this respect, closing price prediction can assist bidders to establish the true value of the item on auction and thus finalise the maximum amount that they are willing to pay. This helps them to develop a bidding strategy to win the auction if the price is appropriate to an item's value, and it also allows experienced bidders to win auctions at a lower cost [13]. By presenting consistent information, closing price prediction supports buyers to make more informed bidding decisions [14]. This also solves some of the information asymmetry problem for buyers, cutting down transaction time and cost. At the same time, sellers can use predictions to identify when the market favours selling their products and assess the value of their inventory better. They can also optimise auction attributes and the selling price for their wares [15].

Predicting the end-price of an online auction is challenging because it depends on auction's attributes which are dynamic in nature $[11,15,16]$. The amount of the winning bid can be forecasted effectively by analysing the data produced as an auction progresses (historical data). Analysis of the plethora of data produced in online auction environments can be done using data mining techniques to predict the end-price of an online auction $[6,17,18]$. Data from a series of identical or similar closed auctions has been used in the past to forecast the winning bid by exploiting regression, classification and regression trees, multi-class classification and multiple binary classification tasks $[11,19]$. The history of an ongoing auction also contains significant information; this can be exploited for short-term forecasting of the next bid by using support vector machines and functional k-nearest neighbor approaches [20], clustering [18] and regression and classification techniques [15].

Furthermore, bidders repeatedly adjust their bids towards their maximum valuation of an item based on the time left in the auction and the bids placed by other participants. This triggers different bidding behaviours. Analysis of these bidding behaviours suggests that agents can be categorised as evaluators, participators, opportunists, snipers, unmaskers or shill bidders [21, 22]. Evaluators have a clear idea of their valuation of the item and place a single, significantly high bid during the early phase of the auction. Participators make a low initial bid and then place ascending bids as the auction progresses. Opportunists place the minimum required bid just before the auction closes. Snipers bid in the closing seconds of the auction. Unmaskers make multiple bids, bidding continuously over a short span of time, without any intermediate bids while the auction is progressing. Shill bidders do not intend to win the auction and place fake bids to increase the end-price of the item. These different types of bidders all perform continuous, early or late bidding based on their bidding behaviours. Late bidding especially has drawn considerable attention from professionals and researchers of eBay-style auctions, which apply hard closing rules with fixed end-times $[1,23]$. The decision of bidders in these environments to postpone their bids until the auction's last moments is indeed a rational and effective winning strategy [24]. This may be the best response to a variety of incremental bidding strategies because late bidders deprive incremental bidders of ample response time; they perform intelligent bidding, drawing on the information they have gathered from the earlier bids of these incremental bidders. Late bidders can also protect their private information about the value of an item, avoiding bidding wars with incremental bidders who compete in these auctions; this leads to higher payoffs for the winners [25].

Nevertheless, there are risks involved with last-second bidding. These late-coming bids may be lost in Internet congestion and extended connection times. As a result, bids may not reach the auction before its closes. One survey found that $86 \%$ of participants had experienced this problem at least once [25]. In addition, a strict focus on calculated last-moment bidding does not allow for the emotional overbidding that most buyers experience when bidding on eBay. Moreover, if we consider the auction-bidding process as a kind of sport, last-moment bidding shows poor sportsmanship that misleads the auction. Therefore, in this article, bidding strategies are designed not only for the types of bidders who interject a single bid at the last second in the auction, but also for those who place one or many bids towards the tail end.

Against this background, the research reported in this article addresses the problem of how to develop successful bidding strategies for the different bidding behaviours of the buyers who take part in eBay auctions. When designing bidding strategies for the eBay environment, there are a number of common challenges that have to be dealt with. Of all of these, predicting the closing prices of ongoing auctions and allowing for the behaviour of different bidders are the most critical concerns for those trying to find optimal bidding strategies for bidding agents. These bidding strategies are designed for potential buyers and aim to forecast their bid amounts at a particular moment in time based on their bidding behaviour and their valuation of an auctioned item.

An Automated Dynamic Bidding Agent-ADBA is developed that uses fuzzy reasoning techniques for bidding in an environment of multiple and simultaneous online auctions of same or similar items. ADBA selects an auction to participate in and assesses the value of the item as in [26]. The final price of the selected auction is predicted by designing bidding strategies based on bidding behavior of bidders using Mamdani's Method for fuzzy relations with regression techniques and Negotiation decision functions. This paper concentrates on designing bidding strategies for the buyers.

The rest of the paper is organised as follows. In section II we present the methodology for developing the biding strategies for the bidding agent. Section III and Section IV design the bidding strategies using fuzzy regression techniques and negotiation decision functions respectively. Section V evaluates the designed methodologies. Section VI depicts experimental results evaluating the success rate and the expected utility of the bidding strategies designed for different bidding behaviors of the bidders. Section VII concludes the paper.

\section{Designing Bidding Strategies For The ADBA AgEnt}

The bidding strategies for ADBA agents are designed based on the calculation of a bid amount at a particular moment in time for bidders who place one or many bids towards the tail end of the auction. 
These bidders are categorised based on the number of bids they make and the timing of their bid placement. Those who make just a single bid are identified as Mystical and Sturdy bidders. Mystical place their only bid in the closing moments (the last five seconds) of the auction while Sturdy bidders lodge just one bid in the five minutes before the end. Bidders who make several bids in the last hour of the auction are identified as Strategic. These bidders up their bid amounts strategically based on the bids recorded by other participants. Generally speaking, bidders tend to exhibit one of two attitudes: they may be desperate to win an item, or they may be willing to bargain for it [27]. These two attitudes are described here as Ambitious and Sophisticated respectively.

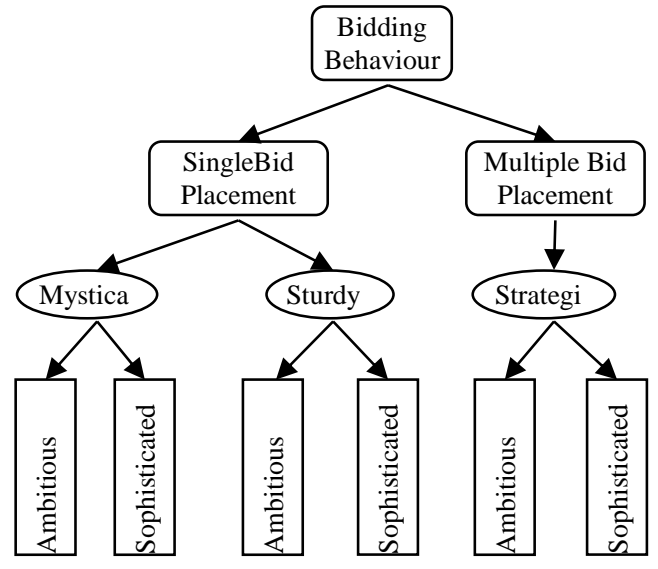

Fig. 1. Types of bidders

A bid by these bidders equals the maximum value that the agent is willing to pay at that point in time. The agent determines this value based on bidding characteristics such as the auction's attributes, the bidder's own attitude and other bidders' attitudes. The auction's attributes have been considered in predicting the initial price of the auction in [26]. Two types of the bidder's own attitudes, Ambitious and Sophisticated, are also taken into account in this study.

Other bidders' attitudes are used to gauge competition in an auction; their previous bids (competing bids) are noted and exploited. Bidders update their bids at a particular moment in time based on others' bids [28]. When the earlier offers of other bidders (competing bids) are higher and the rate of bid change accelerates, a bidder will make higher bids in more frequent increments to win the auction. These are indications of heightened competition. Further, the time left until the auction closes is an important factor that also affects competition. Bidders decide fast towards the end of an auction due to the time pressure. This pressure increases arousal, and bidders bid beyond their limits as the deadline approaches since there is so little time left [29]. This exacerbates the sense of competition among auction participants. In other words, competition rises as the remaining duration of the auction wanes.

The bidding strategies in this article are designed to model the bid amount at a particular moment in time for each bidding behaviour in Fig. 1 using fuzzy reasoning with regression techniques and negotiation decision functions.

\section{BIDDING STRATEGIES USING FUZZY REGRESSION TECHNIQUE}

Mamdani's Method for fuzzy relations and the compositional rule of inference are used to design bidding strategies for buyers [30]. The value of the auction is calculated based on the competition in that auction, and the bidding attitude of buyers with different bidding behaviour (where bid increment $(\Delta P)$ is the amount by which the bidder raises the current bid and the current bid is the initial bid $p_{i}$, the predicted closing price of the selected auction, as described in [26]). The multiple linear regression is used to further improve the auction value. First, the level of competition in the auction is assessed, and then the bid increment is calculated for different bidding behaviours of bidders. Finally, regression models are used to calculate bid amount of the auction.

\section{A. Competition Assessment}

The degree of competition is assessed using the remaining duration of the auction and the previous offers made by competing participants. Assume $C$ is competition, having a fuzzy set of values as $c_{1}, c_{2}, \ldots \ldots \ldots c_{n}, D$ is the remaining duration, having a fuzzy set of values as $d_{1}, d_{2}, \ldots \ldots . d_{n}$ and $\mathrm{B}$ is competing bids, having a fuzzy set of values $b_{1}, b_{2}, \ldots \ldots . . b_{n}$. According to Mamdani's Direct Method, the adaptability $n$ no. of rules $w_{1}, w_{2} \ldots \ldots . w_{n}$ are found as follows:

$$
\begin{aligned}
& w_{1}=\mu d_{1}(D) \quad \square \mu b_{1}(B) \\
& w_{2}=\mu d_{2}(D) \square \mu b_{2}(B) \\
& \ldots \ldots \ldots \ldots \ldots \ldots \ldots \ldots \\
& w_{n}=\mu d_{n}(D) \quad \square \mu b_{n}(B)
\end{aligned}
$$

Competition is then assessed for each rule as follows:

$$
\begin{aligned}
& \mu c^{\prime}{ }_{1}(C)=w_{1} \square \mu c_{1} \\
& \mu c^{\prime}{ }_{2}(C)=w_{2} \square \mu c_{2} \\
& \ldots \ldots \ldots \ldots \ldots \ldots \ldots \ldots \ldots \ldots \ldots \ldots \ldots \ldots \ldots \ldots \ldots
\end{aligned}
$$

These rules are aggregated for the final competition evaluation:

$$
\mu c(C)=\mu c_{1}^{\prime}(C) \wedge \mu c_{2}^{\prime}(C) \wedge \ldots \ldots \ldots \ldots \ldots \ldots \ldots \ldots . . \wedge \mu c_{n}^{\prime}(C)
$$

A definite value of competition is found by applying centre of gravity of the fuzzy set in (1) as follows;

$$
C=\frac{\int \mu c(C) C d C}{\int \mu c(C) d C}
$$

\section{B. Bid Determination}

The bid increment $\Delta P$ for the auction is calculated based on attitudes and competition by applying Mamdani's Method for fuzzy relations and the compositional rule of inference. Let $\Delta P$ have the fuzzy set of values $p_{l}, p_{2}, \ldots \ldots . . p_{n}, E$ is attitudes with a fuzzy set of values as $e_{1}, e_{2} \ldots \ldots \ldots e_{n}$ and $C$ is competition with a fuzzy set of values as $c_{1}, c_{2}, \ldots \ldots . . c_{n}$. 
Here, premise 1: IF $c$ is $C$ and e is E THEN $p$ is $\triangle P$ premise 2: $c$ is $C^{\prime}$ 'and e is $E^{\prime}$ consequence: $p$ is $\Delta P^{\prime}$

where $C, C^{\prime}, E, E$ ', $\Delta P$ and $\Delta P^{\prime}$ are fuzzy sets. As per the mechanism of fuzzy reasoning, we infer " $p$ is $\Delta P^{\prime \prime}$ when the condition " $c$ is $C$ ' and e is $E$ '" is given for the rule " $I F c$ is $C$ and e is $E$ THEN $p$ is $\triangle P^{\prime \prime}$.

Using a fuzzy relations approach, we first convert the $I F$ THEN rule in premise 1 into the fuzzy relation $R_{C}$ and $E \rightarrow \triangle P$. Then, by applying compositional operation, we infer conclusion $\triangle P^{\prime}$ from the fuzzy relations $R_{C}$ and $E \rightarrow \triangle P$ and the condition "c is $C$ ' and e is $E$ " of premise 2 (Fig. 2).

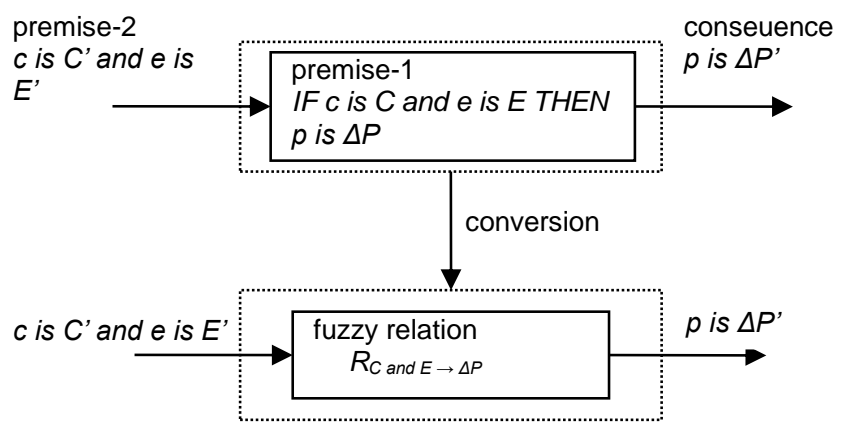

Fig. 2. Fuzzy reasoning by using fuzzy relations and the compositional rule of inference

According to Mamdani's Method for fuzzy relations and the compositional rule of inference, the rule $e_{i}$ and $c_{j} \rightarrow p_{k}$ is described by:

$$
R=\int_{E \times C \times \Delta P} \frac{\left(\mu e_{i}(E) \wedge \mu c_{j}(C) \wedge \mu p_{k}(\Delta P)\right)}{(E, C, \Delta P)}
$$

The conversion in (3) is based on a Cartesian product such as:

$$
e_{i} \text { and } c_{j} \rightarrow p_{k}=e_{i} \wedge c_{j} \wedge p_{k}
$$

The conversion by using the membership value form is given as follows:

$$
\mu R(E, C, \Delta P)=\mu e_{i}(E) \wedge \mu c_{j}(C) \wedge \mu p_{k}(\Delta P)
$$

For $n$ number of rules, the compiled fuzzy relation $R$ is given as:

$$
R=R_{1} \cup R_{2} \cup \ldots \ldots \ldots \ldots R_{n}=\bigcup_{i=1}^{n} R_{i}
$$

For the input of fuzzy sets $E^{\prime}$ on $E$ and $C^{\prime}$ on $C$, the output fuzzy set $\Delta P^{\prime}$ on $\Delta P$ can be obtained as follows:

$$
\Delta P^{\prime}=\left(E^{\prime} \text { and } C^{\prime}\right) \circ R=E^{\prime} \circ\left(C^{\prime} \circ R\right)=C^{\prime} \circ\left(E^{\prime} \circ R\right)
$$

The value for the auction is calculated as $p_{i}+\Delta P^{\prime}$

The fuzzy set $E^{\prime}$ depends on the bidding strategy selected for the bidding agent. Ambitious bidders always have a higher attitude to win the auction than Sophisticated bidders because the Ambitious bidders are desperate to get the item. Accordingly, the fuzzy set $E^{\prime}$ is described such that $E$ is high for Ambitious bidders and low for Sophisticated bidders. However, Sophisticated Strategic bidders have a higher attitude to win the auction than the Mystical and Sturdy bidders of similar type, so $E$ is also high for Sophisticated Strategic bidders.

The auction values are used to predict the bid amount of the auctioned item using multiple linear regression technique. This model has been used to fit a linear relationship between the dependent variable and a set of predictor variables. The multiple linear regression model is employed based on the assessed competition and the calculated auction values. To predict the bid amount, regression coefficients for each predictor variable are opted such that the sum of squares between the predicted and the actual bid over all the training auction data is minimal [26].

$$
\sum_{i=1}^{n}\left(y_{i}-\sum_{j=0}^{m} w_{j} a_{i j}\right)^{2}
$$

where $y_{i}$ is the bid amount of the item for the $i^{\text {th }}$ auction $m$ is the total number of predictor variables $w_{j}$ is the regression coefficient for the $j^{\text {th }}$ predictor variable $a_{i j}$ is the $j^{\text {th }}$ predictor variable for the $i^{\text {th }}$ auction.

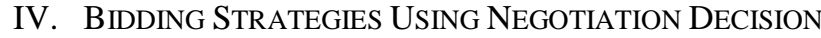 FUNCTIONS}

The bidding strategies are designed by calculating the bid amount at a particular moment in time based on the initial price $\left(p_{i}\right)$ (as in [26]) and using the negotiation decision functions ( $N D F s$ ) given by Faratin et al. [31]. First of all, a bid value is recommended at a particular moment in time based on the competition in the auction. Second, this value is updated to determine the bid amount for buyers with different bidding behaviours.

\section{A. Competition and Bid Determination}

The bid amount is calculated using negotiation decision functions $(N D F s)$ in two phases. In the first phase, the amount is determined based on the competition in an auction. NDFs are applied for the remaining duration of the auction and competing bids. In the second phase, the bid is updated in order to design bidding strategies for each type of bidding behaviour in Fig. 1 according to the listed bidding attitudes.

Assume that $F(t)$ is the function that determines the bid amount based on the remaining duration and $F_{c}(t)$ is the function that determines the bid amount based on competing bids. Assume also that the agent's bids occur at time $0 \leq t \leq$ $t_{\max }$ and the agent's bidding limit is $\left[\min _{b}, \max _{b}\right]$. The bidding 
agent defines a constant $k$, which when multiplied by the size of the interval, gives the value of the starting bid amount. $F(t)$ with $0 \leq t \leq t_{\max }$ is represented using a function $\alpha(t)$ as follows:

$$
F(t)=\min _{b}+\alpha(t)\left(\max _{b}-\min _{b}\right)
$$

where

$$
\alpha(t)=k+(1-k)\left(\min \left(t, t_{\max }\right) / t_{\text {max }}\right)^{1 / \beta}
$$

A wide range of time-dependent functions can be calculated by varying the value of $\alpha(t)$, where $0 \leq \alpha(0) \leq 1, \alpha(0)=k$ and $\alpha\left(t_{\max }\right)=1$, which ensures that the bid amount remains within the value range. Initially this represents the starting bid and at the end of the auction at $t=t_{\max }$, the bid amount reaches the reservation price of bidder, i.e. $p_{r}$ (the maximum value of the auctioned item set by the bidder).

$\beta$ is a constant which belongs to $R^{+}$. A number of possible bidding regulations can be obtained by varying the value of $\beta$ for different bidder-specific issues. For Ambitious bidders who are desperate to have the item, $\beta>1$ and the agent quickly goes to its reservation price $p_{r}$. where $p_{r}=p_{i}=\max _{b}$. The mathematical model for this behaviour is as follows:

$$
\operatorname{Lim}_{\beta \rightarrow+\infty} k+(1-k)\left(\min \left(t, t_{\max }\right) / t_{\max }\right)^{1 / \beta}=1
$$

For Sophisticated bidders, who are willing to bargain for an item, $\beta<1$ and the minimum bid amount is maintained until $t_{\max }$ is almost reached. The mathematical model for this behaviour is as follows:

$$
\operatorname{Lim}_{\beta \rightarrow O^{+}} k+(1-k)\left(\min \left(t, t_{\max }\right) / t_{\max }\right)^{1 / \beta}=k
$$

The computation of $\alpha(t)$ with respect to time (presented here as relative to $t_{\max }$ ) for $\beta \geq 1$ and $\beta \leq 1$ is presented graphically in Fig. 3.

$F_{c}(t)$ computes the bid amount at time $t$ based on the previous bids placed by other participants. To calculate $F_{c}(t)$ at a particular moment of time $t$, the agent reproduces the behaviour of the other participants in earlier steps for $\delta \geq 1$ where $n>2 \delta$.

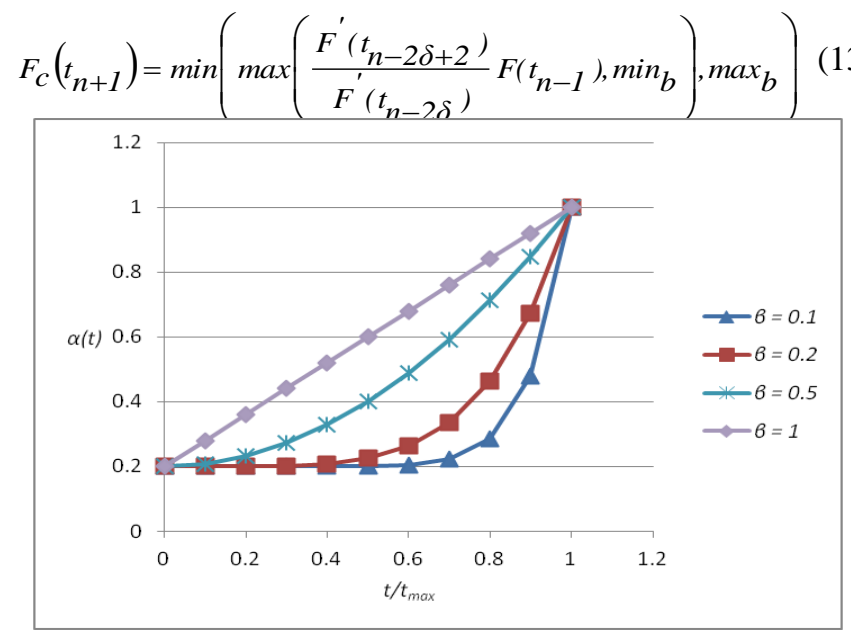

where $F^{\prime}(t)$ is the bid amount placed by the other participants at time $t$.

At a given time, the bidding agent may consider any combination of these issues based on its current situation, As such, bid amounts can be computed to reflect the level of competition in the auction.

(a)

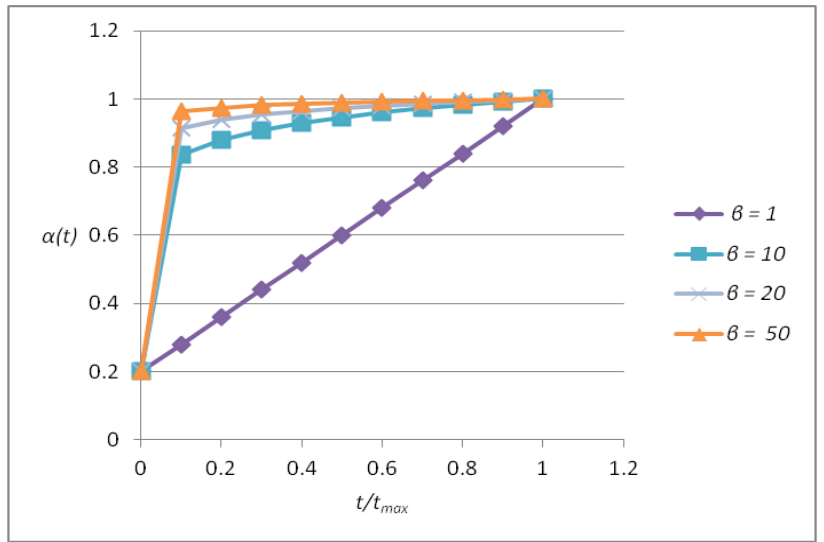

(b)

Fig. 3. Fuzzy Computation of $\alpha(t)$ for $\beta \geq 1$ (a) $\beta \leq 1$ (b)

In the second phase, the bid amount is updated to design bidding strategies for bidders with different bidding behaviours, as detailed below.

\section{1) Mystical Bidding Strategy}

An agent with this strategy places a single bid in the closing moments of the auction. This bid amount depends upon the remaining duration of the auction, as well as on competing bids.

The bid amount at time $t$ for Mystical behaviour will be calculated as the average of $F(t)$ as in (9) and $F_{c}(t)$ in (13). Here, $\min _{b}$ is the lower bound of the bid value at the start of the last five seconds of the auction. The values for $k$ and $\beta$ are set according to the bidding attitude of the bidders. For Ambitious Mystical bidders, the value of $k$ will be high and $\beta>$ 1 , since this type of bidder bids at a price near to the reservation price $p_{r}$. On the other hand, for Sophisticated Mystical bidders, the value of $k$ will be low and $\beta<1$.

2) Sturdy Bidding Strategy

This strategy is similar to Mystical bidding behaviour with the exception of the time at which a bid is placed. A bidder with Sturdy behaviour will place a single bid during the last five minutes of the auction based on the time remaining and the competing bids.

$F(t)$ and $F_{c}(t)$ functions are similar to those in Mystical behaviour where they are used to compute the bid amount. Here, however, $\min _{b}$ is the lower bound of the bid amount at the beginning of the last five minutes of the auction. The values for $k$ and $\beta$ for Ambitious Sturdy and Sophisticated 
Sturdy bidders follow the same conventions as when Mystical bidders have these attitudes.

3) Strategic Bidding Strategy

Strategic bidders place multiple bids during the last hour of an auction. In this strategy, each and every bid is strategically placed based on the bids of competing bidders in the auction. These bidders continue bidding until the bid amount reaches their reservation price $p_{r}$.

Each bid will be calculated as the average of $F(t)$ and $F_{c}(t)$. The value of $\min _{b}$ is the lower bound of the bid amount at the beginning of the last hour of the auction. An Ambitious Strategic bidder starts bidding at a value close to his valuation for the item; the values for $k$ are high for this bidding strategy. Here $\beta>1$, since bidders with an Ambitious attitude tend to quickly reach $p_{r}$ before the deadline is reached by placing multiple bids. However, Sophisticated Strategic bidders do not start bidding at an amount close to $p_{r}$; rather, they increase their bid amount slowly based on the other bids in the auction. As such, the values for $k$ are low and $\beta<1$ for Sophisticated Strategic behaviour.

TABLE I

CHOICE OF $K$ AND $\beta$ FOR DIFFERENT BIDDING STRATEGIES

\begin{tabular}{|c|c|c|}
\hline Bidding Strategy & $\boldsymbol{k}$ & $\boldsymbol{\beta}$ \\
\hline AMST & $0.6 \leq \mathrm{k} \leq 1$ & $\beta>1$ \\
\hline SMST & $0 \leq \mathrm{k} \leq 0.3$ & $\beta<1$ \\
\hline ASTD & $0.6 \leq \mathrm{k} \leq 1$ & $\beta>1$ \\
\hline SSTD & $0 \leq \mathrm{k} \leq 0.3$ & $\beta<1$ \\
\hline ASTG & $0.6 \leq \mathrm{k} \leq 1$ & $\beta>1$ \\
\hline SSTG & $0 \leq \mathrm{k} \leq 0.6$ & $\beta<1$ \\
\hline
\end{tabular}

Assume AMST and SMST represent the Ambitious Mystical and Sophisticated Mystical bidding strategies respectively, and that ASTD and SSTD represent the Ambitious Sturdy and Sophisticated Sturdy bidding strategies respectively. ASTG and SSTG represent the Ambitious Strategic and Sophisticated Strategic bidding strategies respectively. The values of $k$ and $\beta$ for these behaviours are shown in Table I.

\section{Evaluating Bidding StRATEgIES}

The bidding strategies based on the NDFs and fuzzy reasoning were evaluated separately by performing two sets of experiments. First, the NDFs based bidding strategies will be evaluated.

The ADBA system generates two types of agents for evaluating the bidding strategies based on NDFs: NDF-DC and NDF-D. The NDF-DC agents design the bidding strategies using the $N D F s$, which depend on both the $F(t)$ and
$F_{c}(t)$. Here $D C$ refers to the agents that calculate the bid amount using the remaining Duration of the auction and Competing bids. NDF-D agents design bidding strategies using $N D F s$, which only depend on $F(t)$ and not on $F_{c}(t)$. Here $D$ refers to agents that calculate the bid amount using the remaining Duration of the auction. In order to evaluate the performance of both $N D F-D C$ and NDF-D agents in a wide variety of test environments, the agents were subjected to different action settings and to different bidding restrictions (bargain level). In this set of experiments, to compute $F(t)$, values for $k$ and $\beta$ were chosen as given in Table I. To compute the function $F_{c}\left(t_{n+1}\right)$, the initial values of $F^{\prime}\left(t_{n-2 \delta+2}\right), F^{\prime}\left(t_{n-2 \delta}\right)$ and $F\left(t_{n-1}\right)$ were calculated at $\delta=1$ for all the bidding strategy types i.e. Mystical, Sturdy and Strategic. For Mystical, Sturdy and Strategic behaviours, $\delta=1$ at the beginning of the last five seconds, the last five minutes and the last hour of the auction respectively. Initial values of $F^{\prime}\left(t_{n-2 \delta+2}\right), F^{\prime}\left(t_{n-2 \delta}\right)$ and $F\left(t_{n-1}\right)$ were assigned from the bid history of the auction in which the bidders were then participating. The current maximum bid was assigned to $F^{\prime}\left(t_{n-2 \delta+2}\right)$, and the previous bid was assigned to $F\left(t_{n-1}\right)$ followed by $F^{\prime}\left(t_{n-2 \delta}\right)$.

Two types of attitudes of bidders were considered: Ambitious and Sophisticated. Bidders with an Ambitious attitude start bidding at a higher price close to their reservation value $p_{r}$ and their bid amount is not so affected by the bid amounts placed by the other bidders due to their desperate behavior. AMST, ASTD and ASTG bidding strategies follow this type of attitude. Bidders who are willing to bargain always bid strategically based on the bids placed by the other competitors. SMST SSTD and SSTG follow this type of attitude. As the bidding strategies described above select bids based on the remaining time as well as on the bids placed by the other participants, these strategies will be successful when the bidder has a desire to bargain attitude. Thus, we need to evaluate the performance of agents who act strategically based on the bids placed by the other participants, i.e. bidders with a Sophisticated attitude.

In the second set of experiments, Fuzzy agents were evaluated against the $N D F-D C$ agents in a similar auction environment as above. In order to compute $\Delta P$, linguistic variables for the bidder's attitude and competition assessment were chosen. The bidding strategies were analysed by considering the following sets of logical rules using various fuzzy sets:

Rule 1: IF the attitude of the agent to winning the auction is $E_{l}$ AND competition on the market for that product is $C_{l}$, THEN the bid increment will be $P_{l}$

Rule 2: IF the attitude of the agent to winning the auction is $E_{1}$ AND competition on the market for that product is $C_{2}$, 
THEN the bid increment will be $P_{2}$

Rule 3: IF the attitude of the agent to winning the auction is $E_{2}$ AND competition on the market for that product is $C_{l}$, THEN the bid increment will be $P_{2}$

Rule 4: IF the attitude of the agent to winning the auction is $E_{2}$ AND competition on the market for that product is $C_{2}$, THEN the bid increment will be $P_{3}$

These fuzzy sets represent the linguistic variables as follows: attitudes low as $E_{l}$ and high as $E_{2}$, competition low as $C_{l}$ and high as $C_{2} . P_{1}, P_{2}$ and $P_{3}$ were the bid increments based on the characteristics of the auction, where $P_{3} \geq P_{2} \geq P_{1}$. We assumed that the set of attitudes for buying any item was $E=\left[e_{1}, e_{2}, e_{3}\right]=[0,0.5,1.0]$, and the set of competition for the item on the market was $C=\left[c_{1}, c_{2}, c_{3}\right]=[0,0.5,1.0]$. The fuzzy sets used in the preceding four rules can be quantized as shown in Fig. 4.

$$
\begin{array}{lll}
E_{1}=[1.0,0.5,0] & C 1=[1.0,0.5,0] & P_{1}=[1.0,0,0] \\
E_{2}=[0,0.5,1.0] & C 2=[0,0.5,1.0] & P_{2}=[0,1.0,0] \\
& & P_{3}=[0,0,1.0]
\end{array}
$$
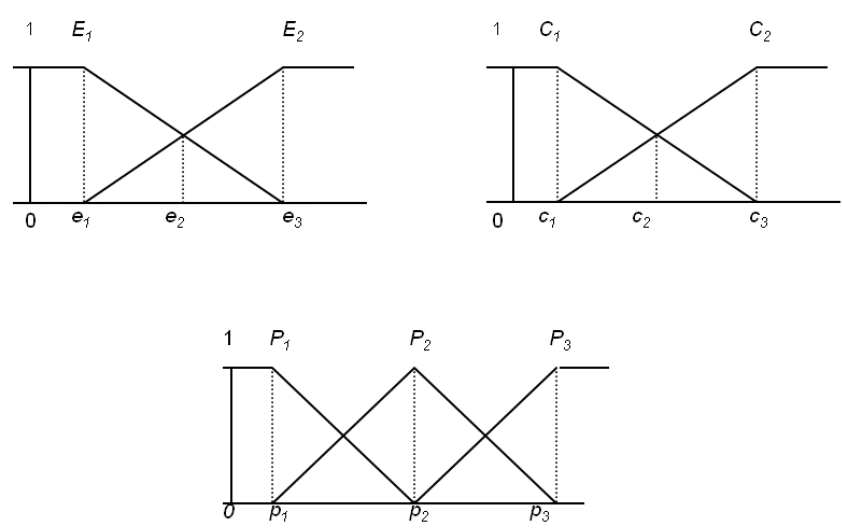

Fig. 4. Fuzzy sets for bidding logic

For all these bidding strategies, competition was assessed based on the remaining duration and the bids placed by other participants (competing bids). These bidding strategies will be successful when the bidder has a desire to bargain attitude, in a similar vein to the situation with the bidding strategies using $N D F s$. This set of experiments, thus, needed to address the performance of bidding agents with a Sophisticated attitude who act strategically based on the bids placed by the other participants.

The success rate percentage and the expected utility of the bidding agents are used as the performance measures.

Success rate percentage $=R_{\text {success }}=r_{\text {success }} * 100$

where $r_{\text {success }}$ is the success rate and $r_{\text {success }}=N_{\text {win }} / N_{\text {total }}$, $N_{\text {win }}$ is the number of auctions won by the agent and $N_{\text {total }}$ is the total number of auctions.

Expected utility $=U_{\text {exp }}=U_{\text {win }} * r_{\text {success }}$

where $U_{\text {win }}$ is the utility of the winning agent.

and $U_{\text {win }}=\left(p_{r}-v_{i}\right) / p_{r}$, where $p_{r}$ is the reservation price (maximum amount the bidder is willing to pay) and $v_{i}$ is the winning price of the auction.

A simulated electronic marketplace was developed and experiments were conducted separately for heterogeneous and homogeneous bidding agents when assessing the bidding strategies based on negotiation decision functions and fuzzy reasoning techniques. In this research, heterogeneous bidders have random or varying willingness-to-bargain levels, here called 'bargain levels'. In contrast, all homogeneous bidders have identical bargain levels.

These bidding strategies were assessed separately in different settings, i.e. low-, medium- and high- bid rate auctions for each bidding agent acting for the heterogeneous bidders. NDF-D and NDF-DC agents with varying bidding strategies competed against one another for each type of auction separately.

Bidding agents with various bidding strategies participated in each type of auction separately. Experiments were run 50, 100,150 and 200 times to test and verify the statistical significance of the results. These experiments were also carried out separately for each type of homogeneous bidding agent in the different bidding environments, in auctions with various bid rates. To evaluate the bidding strategies, the $U_{\text {exp }}$ of NDF-D and NDF-DC bidding agents was measured in two situations: firstly, against the various bid rates of the auctions, and secondly, against the different bargain levels of bidders.

The fuzzy bidding strategies were assessed for heterogeneous bidding agents in different bidding environments, i.e. auctions of the various bid rates. Fuzzy and $N D F-D C$ agents with various bidding strategies competed against one another separately in each type of auction. Again, the experiments were run several times to test and verify the success rate and expected utility of these bidding agents.

\section{EXPERIMENTS}

A simulated electronic marketplace was set up to implement the ADBA agents and thus demonstrate the performance of the bidding strategies.

\section{A. Market Architecture}

In this marketplace buyers negotiate using different bidding strategies rooted in their attitudes, all with the aim of winning an auction. The electronic auction market is managed by an auction server and can be used by various buyers. The auction server is implemented at the server end with Administrator agent and it receives information from Initial Price Estimator agent and auction database. Bidders are entered into the system at the client end using ADBA Bidder agents.

The Initial Price Estimator agent searches for a target auction by assessing the value of the auctioned item using the clustering approach and bid mapping and selection technique [26]. This agent is also responsible for providing information about the target auction to the Administrator agent.

The Administrator agent maintains the auction information provided by the Initial Price Estimator agent. Whenever bidder registers with the Administrator agent to buy an item in an auction, the Administrator agent creates an ADBA Bidder 


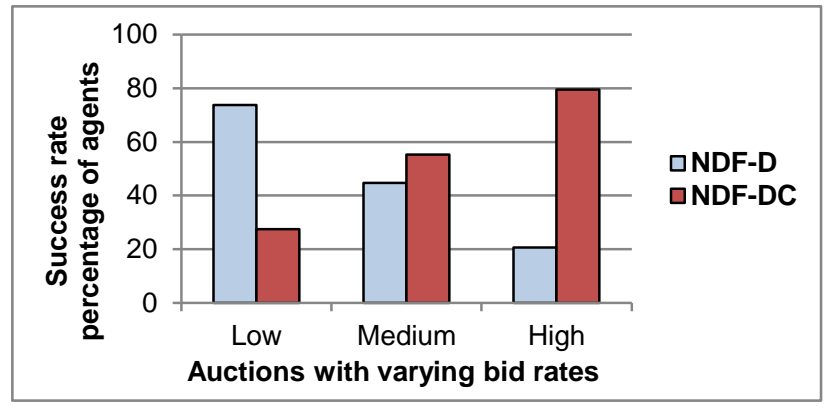

(a)

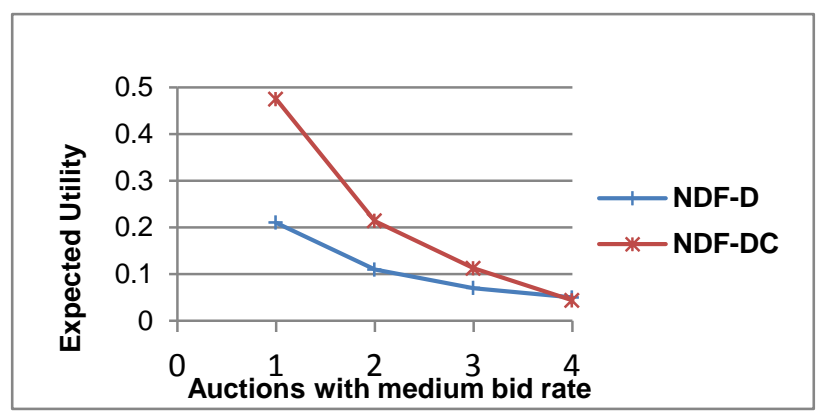

(c)

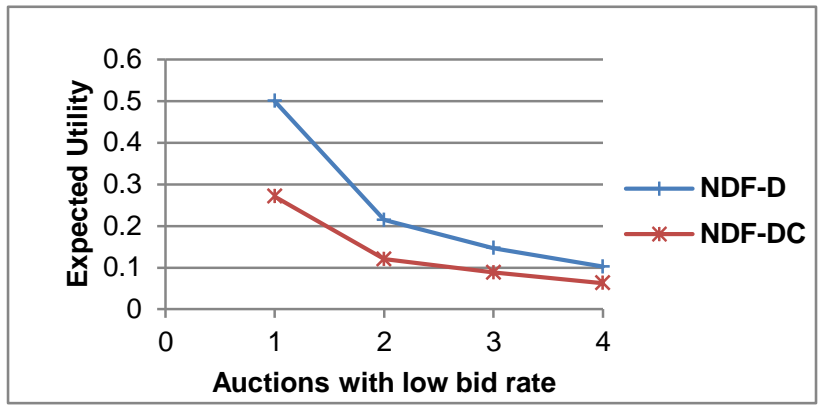

(b)

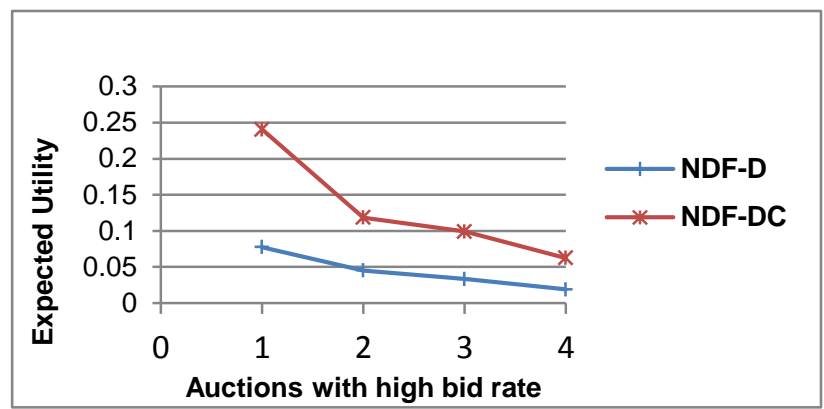

(d)

Fig. 6. Success rate and Expected utility comparison for Mystical behavior

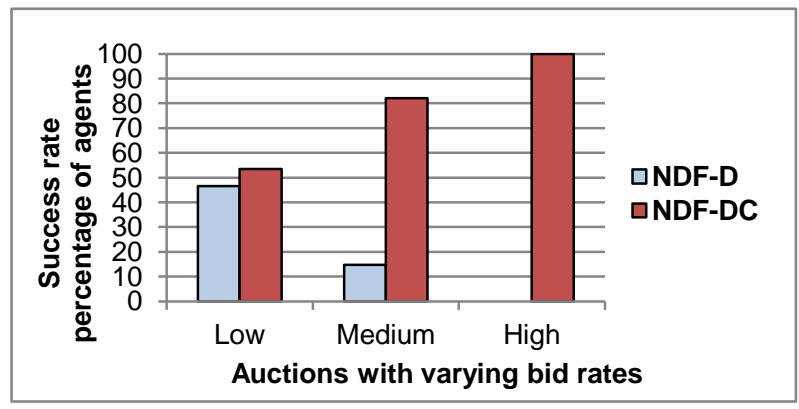

(a)

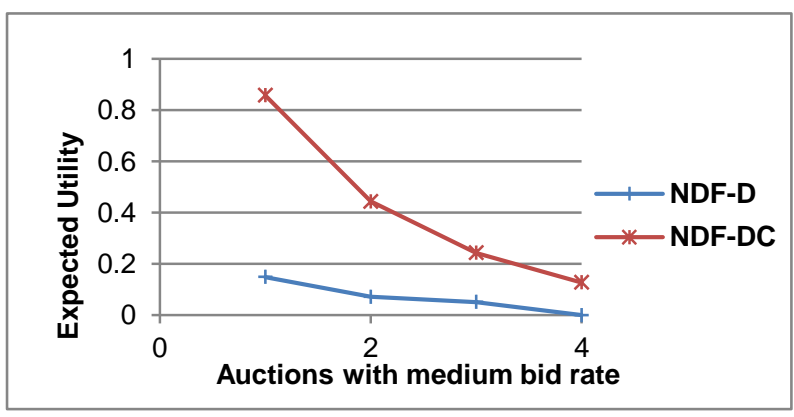

(c)

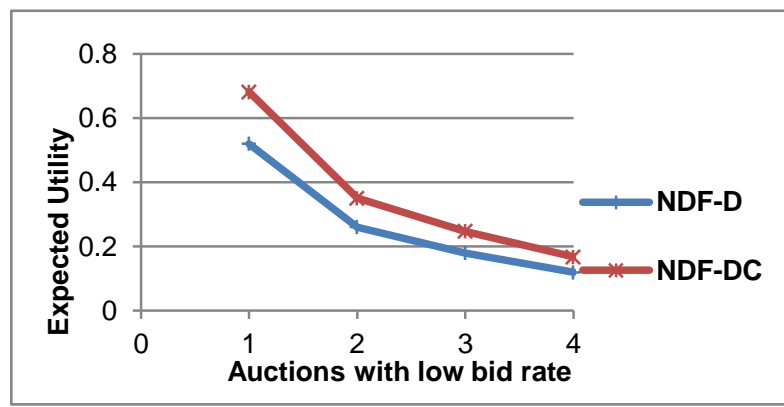

(b)

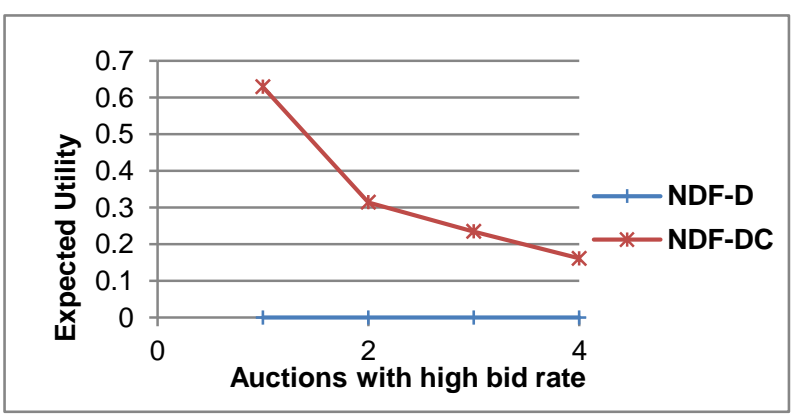

(d)

Fig. 7 Success rate and Expected utility comparison for Sturdy behavior 


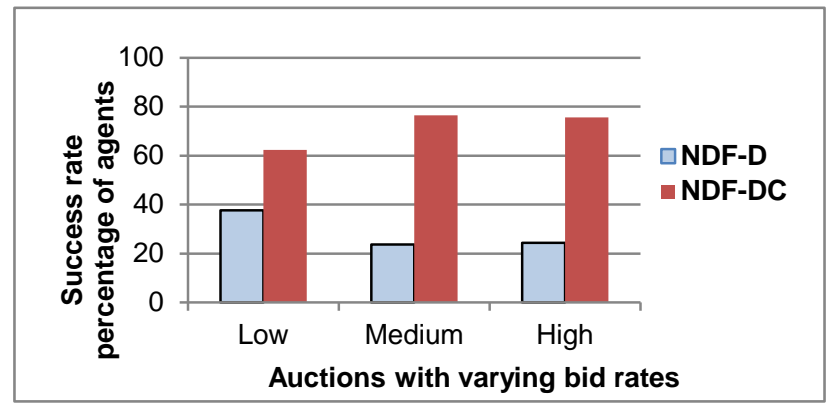

(a)

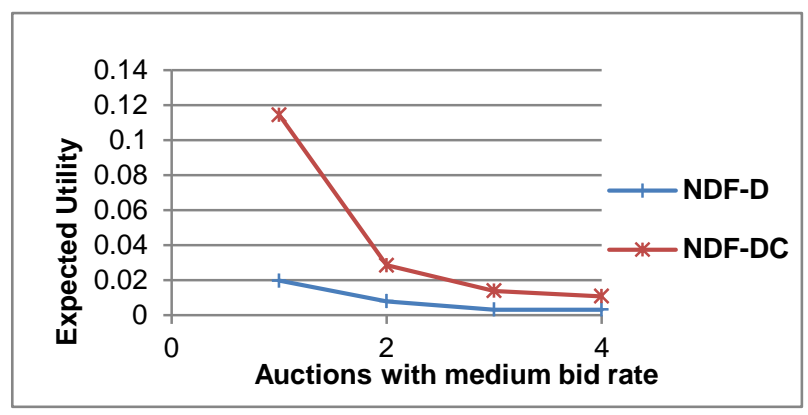

(c)

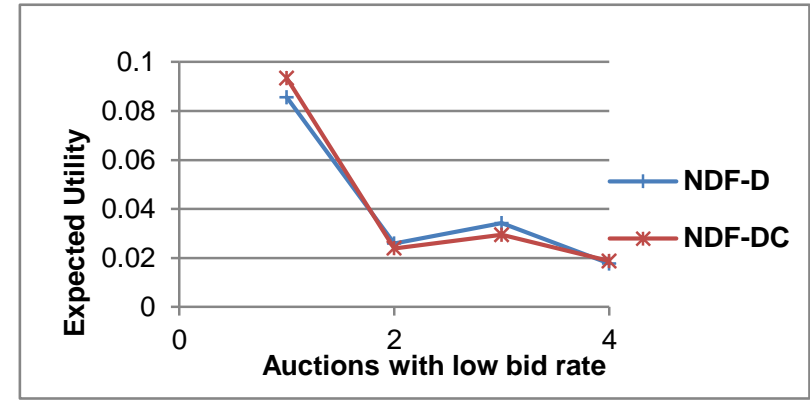

(b)

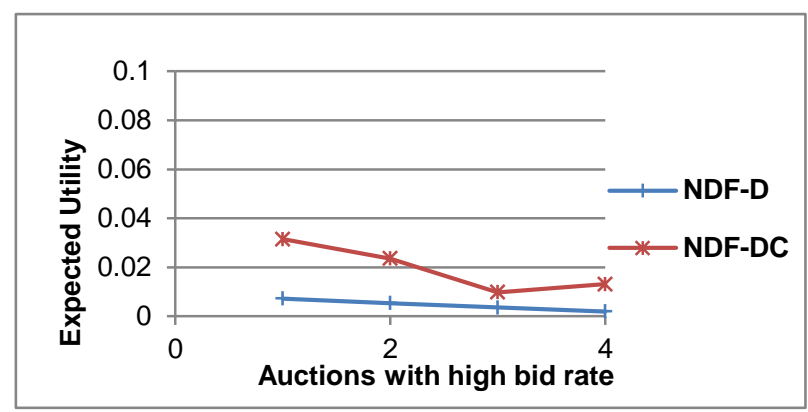

(d)

Fig. 8. Success rate and expected utility comparison for Strategic bidders

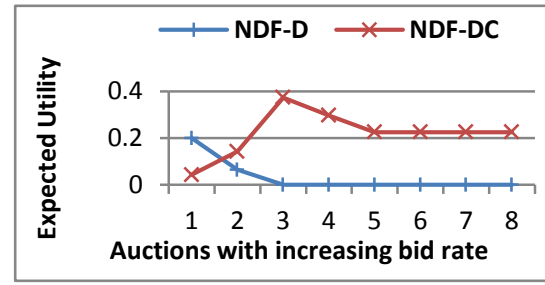

(a)

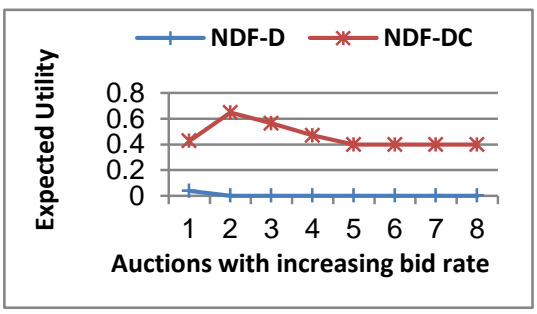

(b)

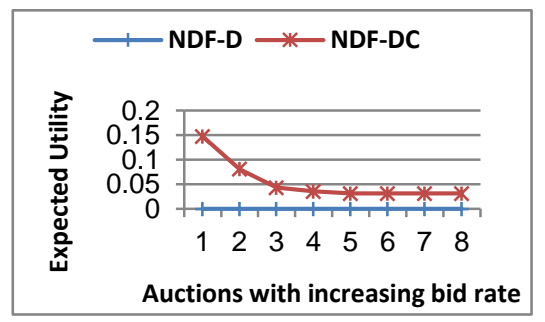

(c)

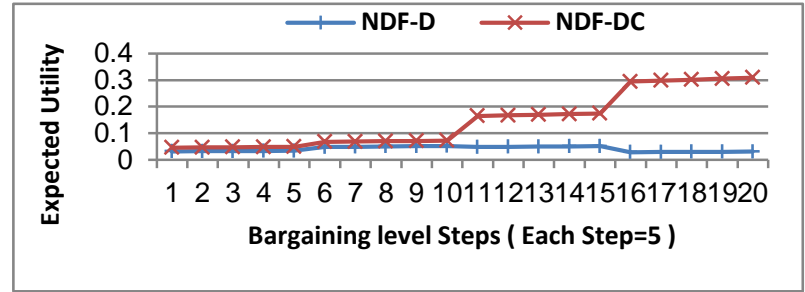

(d)

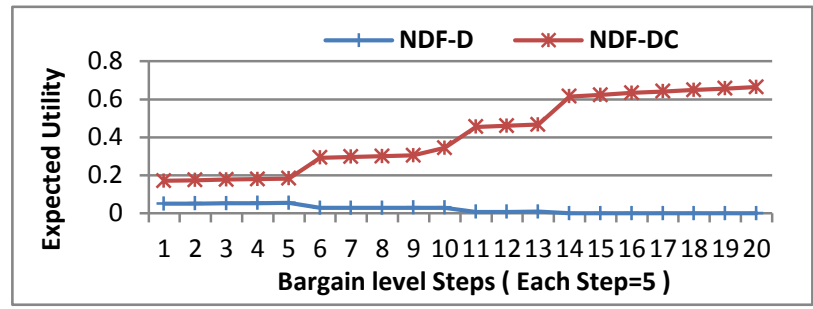

(e)

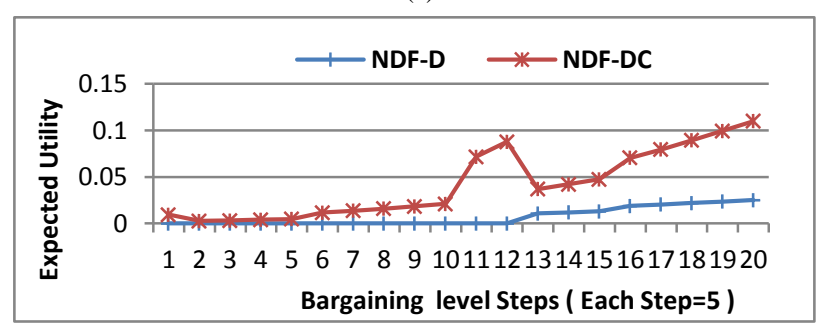

(f)

Fig 9. Experiments for homogeneous bidders. Expected utility comparison for Mystical bidders in (a), Sturdy bidders in (b) and Strategic bidders in (c) with respect to bid rate. Expected utility comparison for Mystical bidders in (d), Sturdy bidders in (e) and Strategic bidders in (f) with respect to their bargain level. 
The auctions in each set of experiments were arranged according to the bid amounts approaching the closing price of the auction. From the graphed results, it is evident that expected utility decreases as auctions approach the closing price. This is in line with expectations.

$R_{\text {success }}$ of $N D F-D C s$ is clearly higher than that of NDF-Ds when these heterogeneous agents with Sturdy behaviour compete in low-, medium- and high- bid-rate-auctions (Fig. 7(a)). Similarly, $U_{\text {exp }}$ of these $N D F-D C s$ is also higher than that of NDF-Ds competing in low-, medium- and high- bidrate auctions (Fig. 7(b) to Fig. 7(d)). In the high-bid-rate settings, $R_{\text {success }}$ and $U_{\text {exp }}$ of the $N D F_{-} D s$ are zero. This corresponds with the intuition that the bid increments made by these $N D F \_D s$ are always lower than those of the $N D F-D C s$. Agents with Sturdy behaviour place bids at the beginning of the last five minutes of the auction, i.e. at a time-point when agents are under less pressure to reach $p_{r}$ than they will be near the final moments of the auction. However, the NDF-DC agents' consideration of competing bids accelerates their own bidding when the high bid rate of an auction raises bid amounts.

$N D F-D C$ agents excel compared with $N D F-D$ agents in terms of $R_{\text {success }}$ when we look at heterogeneous agents with Strategic behaviour competing across low-, medium- and high- bid-rate auction settings (Fig 8(a)). The $U_{\text {exp }}$ of $N D F$ $D C s$ is also higher than that of $N D F-D s$ when these agents compete in medium- and high-bid-rate auctions (Fig. 8(c) and Fig. 8(d)). However, in auctions with low bid rates, the $U_{\text {exp }}$ of the $N D F-D s$ and that of $N D F-D C s$ overlap with one another (Fig. 8(b)). Bidding agents with Strategic behaviour place bids throughout the last hour of the auction, and these bids approach $p_{r}$ slowly for both $N D F-D$ and $N D F-D C$ agents.

The NDF-DC agents' consideration of others' bids hardly affects their own bid increments in these low-bid-rate settings since all the participants' increments are approximately the same as those of NDF-Ds due to their Strategic behaviour.

The experiments were carried out separately for each type of the homogeneous bidder agents across various bidding environments, i.e. auctions with different bid rates. The $U_{\text {exp }}$ of the bidder agents was measured in two situations: firstly, against the various bid rates of the auctions and secondly, against the different bargain levels of the bidders.

The results of these experiments showed the following: Across auctions of different bid rates, homogeneous NDFDCs with Sturdy behaviour and NDF-DCs with Strategic behaviour always achieve higher $U_{\text {exp }}$ than their respective $N D F$-D equivalents (Fig. 9(b) and Fig. 9(c)). In the case of agents with Mystical behaviour, NDF-DCs outperform NDF$D s$ when they compete in medium-to high bid-rate auctions; in low-bid-rate auctions, however, these $N D F-D s$ achieve higher $U_{\text {exp }}$ than the NDF-DCs do (Fig 9(a)). This corresponds with an intuition similar to the one about the heterogeneous bidders. In low-bid-rate auctions, NDF-DCs with Mystical behaviour have limited bid increments. The agents with Mystical behaviour place bids in the closing five seconds of an auction at the point when all participants' bids approach $p_{r}$. However, the NDF-DC agents' consideration of others' bids reduces their bid increments when the low bid rate decreases the amounts that other participants bid.
The $U_{\text {exp }}$ of homogeneous $N D F$-based bidding agents was also measured against their different bargain levels varying from 0 to 100 in steps of 5 . Here $U_{\text {exp }}$ represents the average of expected utilities of bidding agents in auctions with various bid-rates. The results showed that the NDF-DCs with Mystical, Sturdy and Strategic behaviours always achieved higher $U_{\text {exp }}$ than the counterpart NDF-Ds of each of these types (Fig. 9(d), Fig. 9(e) and Fig. 9(f)).

In the second set of experiments, Fuzzy bidding agents were evaluated against $N D F-D C$ bidding agents in an auction environment similar to that described above for heterogeneous bidders. For each rule given in Section V, fuzzy relations $(R 1$, $R 2, R 3$ and $R 4$ ) were constructed using Mamdani's method for fuzzy relations and compositional rule of inference. $\mathrm{R} 1$ is shown as in Fig. 10 and the total fuzzy relation $\mathrm{R}$ is given as in Fig. 11.

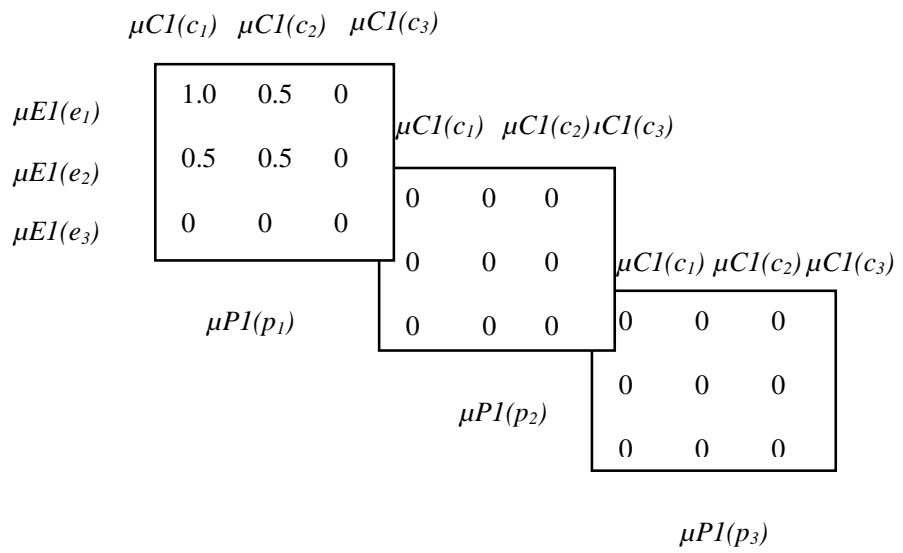

Fig. 10. Fuzzy relation for the fuzzy rule R1

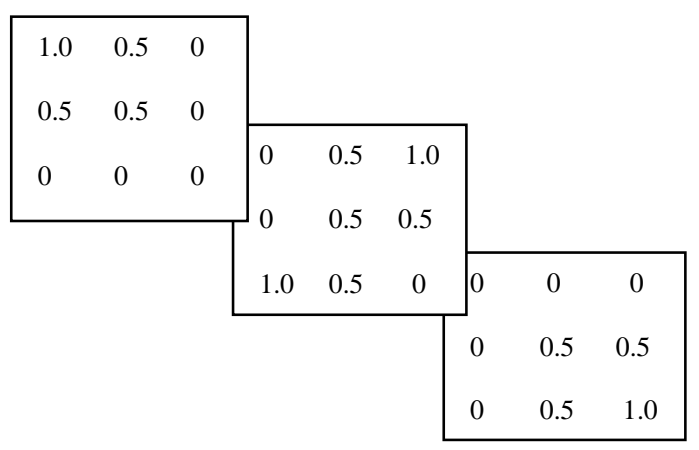

Fig. 11. Total fuzzy relation $\mathrm{R}$ 
The output fuzzy set $\Delta P^{\prime}$ on $\Delta P$ was calculated for different bidding strategies of bidders using input fuzzy sets $E^{\prime}$ on $E$ and $C^{\prime}$ on $C$ by applying Mamdani's compositional rule of inference for different levels of competition (low and high). A definite value of the bid increment was calculated by defuzzifying $\Delta P^{\prime}$ using a centre of gravity with the weighted mean method. The actual value of the bid amount is calculated using multiple linear regression approach. The experiments were carried out for each type of heterogeneous bidder agents separately in different auction environments with various bid rates. $R_{\text {success }}$ and $U_{\text {exp }}$ of the bidder agents were averaged over auctions with various bid rates for each type of heterogeneous bidder. The results are clear in Fig. 12 and Fig. 13. The Fuzzy agents with Mystical, Sturdy and Strategic behaviour outperform their NDF-DCs counterparts of their same behavioural types with respect to $R_{\text {success }}$ and $U_{\text {exp }}$.

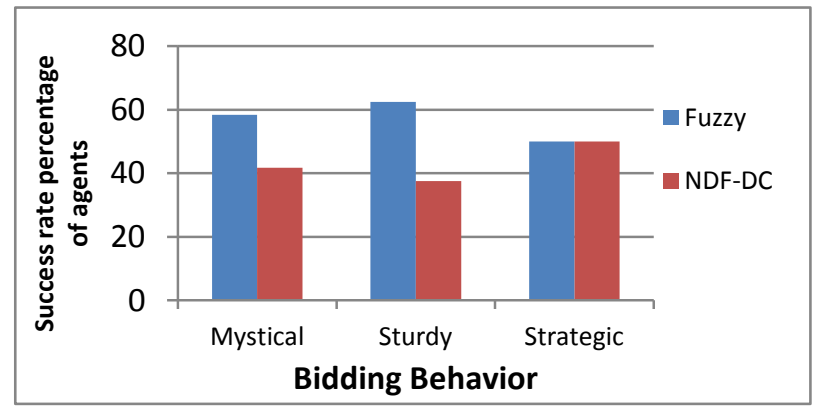

Fig.12. Success rate comparison for Fuzzy and NDF-DC agents

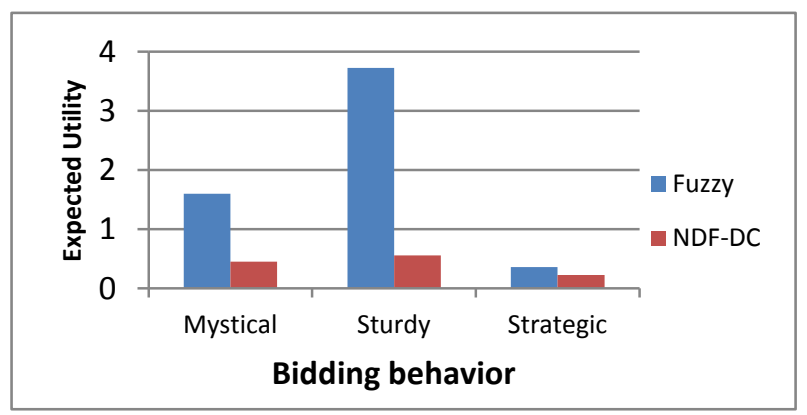

Fig.13. Expected utility comparison for Fuzzy and NDF-DC agents

\section{CONCLUSION}

In this paper we presented a design of bidding strategies for buyers based on their different bidding behaviors. Bidding strategies have been designed that emphasise bidding characteristics such as an auction's attributes, the bidder's own attitude to winning the auction and other bidders' 's behavior. The design has drawn on Mamdani's Method for fuzzy relations and the compositional rule of inference; it has also invoked time- and behaviour- dependent negotiation decision functions. The performance of the heterogeneous and homogeneous bidders following the bidding strategies designed were then measured separately across wide-ranging test environments subject to different auction settings and bidding restrictions. The results demonstrate that bidding agents who adopt the fuzzy regression based bidding approach outperform agents following the methodology of Negotiation Decision Functions in terms of success and expected utility across most settings.

\section{REFERENCES}

[1] A. Ockenfels, D. H. Reiley, and A. Sadrieh, "Online auctions," in Economics and Information Systems Handbook, ed. Amsterdam: Elsevier Science, 2006, pp. 571-622.

[2] E. Haruvy and P. T. L. Popkowski Leszczyc, "Internet Auctions," Foundations and Trends ${ }^{\circledR}$ in Marketing, vol. 4, pp. 1-75, 2010.

[3] Y. H. Park and E. T. Bradlow, "An integrated model for bidding behavior in Internet auctions: Whether, who, when, and how much," Journal of Marketing Research, vol. 42, pp. 470-482, 2005.

[4] W. Jank and S. Zhang, "An automated and data-driven bidding strategy for online auctions," INFORMS Journal on computing, vol. 23, pp. 238-253, 2011.

[5] A. Byde, C. Preist, and N. Jennings, "Decision procedures for multiple auctions," in Proceedings of the first international joint conference on Autonomous agents and multiagent systems: part 2, 2002, pp. 613-620.

[6] W. Jank and G. Shmueli, "Profiling price dynamics in online auctions using curve clustering," Smith School of Business, University of Maryland, Working paper2005.

[7] P. Bajari and A. Hortacsu, "The winner's curse, reserve prices, and endogenous entry: empirical insights from eBay auctions," RAND Journal of Economics, pp. 329-355, 2003.

[8] R. Bapna, P. Goes, and A. Gupta, "Analysis and design of business-to-consumer online auctions," Management Science, vol. 49, pp. 85-101, 2003.

[9] A. E. Roth and A. Ockenfels, "Last-minute bidding and the rules for ending second-price auctions: Evidence from eBay and Amazon auctions on the Internet," The American Economic Review, vol. 92, pp. 1093-1103, 2002.

[10] R. T. Wilcox, "Experts and amateurs: The role of experience in Internet auctions," Marketing Letters, vol. 11, pp. 363-374, 2000.

[11] D. Van Heijst, R. Potharst, and M. Van Wezel, "A support system for predicting eBay end prices," Decision Support Systems, vol. 44, pp. 970-982, 2008.

[12] I. Raykhel and D. Ventura, "Real-time automatic price prediction for ebay online trading," in Proceedings of the Innovative Applications of Artificial Intelligence Conference, 2009, pp. 135140.

[13] E. Sun, "The effects of auctions parameters on price dispersion and bidder entry on eBay: a conditional logit analysis," working paper, Stanford University2005.

[14] I. Raykhel and D. Ventura, Real-time automatic price prediction for ebay online trading: Brigham Young University. Dept. of Computer Science, 2008.

[15] L. Xuefeng, L. Lu, W. Lihua, and Z. Zhao, "Predicting the final prices of online auction items," Expert Systems with Applications, vol. 31, pp. 542-550, 2006

[16] D. Lucking Reiley, D. Bryan, N. Prasad, and D. Reeves, "Pennies from eBAy: The Determinants of Price in Online Auctions," The Journal of Industrial Economics, vol. 55, pp. 223-233, 2007.

[17] V. Nikolaidou and P. A. Mitkas, "A sequence mining method to predict the bidding strategy of trading agents," in Agents and Data Mining Interaction, ed Berlin Heidelberg: Springer, 2009, pp. 139151.

[18] D. D. Kehagias and P. A. Mitkas, "Efficient E-Commerce Agent Design Based on Clustering eBay Data," in International Conferences on Web Intelligence and Intelligent Agent Technology Workshops, 2007 IEEE/WIC/ACM 2007, pp. 495-498.

[19] R. Ghani and H. Simmons, "Predicting the end-price of online auctions," in Proceedings of the International Workshop on Data Mining and Adaptive Modelling Methods for Economics and Management, Pisa, Italy, 2004.

[20] S. Zhang, W. Jank, and G. Shmueli, "Real-time forecasting of online auctions via functional K-nearest neighbors," International Journal of Forecasting, vol. 26, pp. 666-683, 2010. 
[21] R. Bapna, P. Goes, A. Gupta, and Y. Jin, "User heterogeneity and its impact on electronic auction market design: An empirical exploration," Mis Quarterly, vol. 28, pp. 21-43, 2004.

[22] J. Trevathan and W. Read, "Detecting shill bidding in online English auctions," Handbook of Research on Social and Organizational Liabilities in Information Security, pp. 446-470, 2009.

[23] L. Du, Q. Chen, and N. Bian, "An empirical analysis of bidding behavior in simultaneous ascending-bid auctions," in International Conference on E-Business and E-Government (ICEE), , 2010, pp. 249-251.

[24] D. Vergano, "On eBay, It Pays to Snipe," USA Today, vol. 6, 2006.

[25] A. Ockenfels and A. E. Roth, "The timing of bids in internet auctions: Market design, bidder behavior, and artificial agents," $A I$ magazine, vol. 23, p. 79, 2002.

[26] P. Kaur, M. Goyal, and J. Lu, "A price prediction model for online auctions using fuzzy reasoning techniques," in Fuzzy Systems (FUZZ-IEEE), 2014 IEEE International Conference on, 2014, pp. 1311-1318.

[27] P. Anthony and N. R. Jennings, "Developing a bidding agent for multiple heterogeneous auctions," ACM Transactions on Internet Technology (TOIT), vol. 3, pp. 185-217, 2003.

[28] D. Ariely and I. Simonson, "Buying, bidding, playing, or competing? Value assessment and decision dynamics in online auctions," Journal of Consumer Psychology, vol. 13, pp. 113-123, 2003.

[29] G. Ku, D. Malhotra, and J. K. Murnighan, "Towards a competitive arousal model of decision-making: A study of auction fever in live and Internet auctions," Organizational Behavior and Human Decision Processes, vol. 96, pp. 89-103, 2005.

[30] K. Tanaka and T. Niimura, An introduction to fuzzy logic for practical applications: Springer New York, 1997.

[31] P. Faratin, C. Sierra, and N. R. Jennings, "Negotiation decision functions for autonomous agents," Robotics and Autonomous Systems, vol. 24, pp. 159-182, 1998. 